\title{
Hamilton-Jacobi flows and characterization of solutions of Aronsson equations
}

\author{
PETRi JUUTINEN AND EERO SAKSMAN
}

\begin{abstract}
In this note, we verify the conjecture of Barron, Evans and Jensen [3] regarding the characterization of viscosity solutions of general Aronsson equations in terms of the properties of associated forward and backwards HamiltonJacobi flows. A special case of this result is analogous to the characterization of infinity harmonic functions in terms of convexity and concavity of the functions $r \mapsto \max _{y \in B_{r}(x)} u(y)$ and $r \mapsto \min _{y \in B_{r}(x)} u(y)$, respectively.
\end{abstract}

Mathematics Subject Classification (2000): 35J60 (primary); 49L25, 35F25 (secondary).

\section{Introduction}

The problem of minimizing the supremum functional

$$
S(u, \Omega)=\underset{x \in \Omega}{\operatorname{ess} \sup } H(D u(x))
$$

has as an Euler-Lagrange equation the so-called Aronsson equation

$$
A_{H}[u]:=\sum_{i, j=1}^{n} H_{p_{i}}(D u) H_{p_{j}}(D u) u_{i j}=\left(D^{2} u D H(D u)\right) \cdot D H(D u)=0,
$$

see $[4,6]$. Here, and throughout the paper, we assume that the Hamiltonian $H$ : $\mathbb{R}^{n} \rightarrow \mathbb{R}$ satisfies the following conditions:

(i) $H \in C^{2}\left(\mathbb{R}^{n} \backslash\{0\}\right) \cap C^{1}\left(\mathbb{R}^{n}\right), H(0)=0$ and $H(p) \geq 0$ for all $p \in \mathbb{R}^{n}$,

(ii) $H$ is convex and locally uniformly convex outside origin, i.e. for any compact $K \subset \mathbb{R}^{n} \backslash\{0\}$ there exists a constant $c=c(K)>0$ such that

$$
\frac{1}{2}\left(H(p)+H\left(p^{\prime}\right)\right) \geq H\left(\frac{p+p^{\prime}}{2}\right)+\frac{c}{2}\left|p-p^{\prime}\right|^{2}
$$

for all $p, p^{\prime} \in K$.

The first author is supported by the Academy of Finland project 108374.

Received September 29, 2006; accepted January 19, 2007. 
(iii) $H$ is superlinear:

$$
\liminf _{|p| \rightarrow \infty} \frac{H(p)}{|p|}=\infty
$$

Observe that e.g. the Hamiltonian $H(p)=\frac{1}{q}|p|^{q}$ satisfies the above requirements, assuming that $q \in(1, \infty)$. For this kind of very special choices, where $H$ depends only on the modulus $|p|$, the equation (1.1) is equivalent to the popular infinity Laplace equation, first investigated by Aronsson [1]. It governs the problem of finding "optimal" Lipschitz extensions and appears in many other applications, see [2]. Recently the infinity Laplace equation has been given a game-theoretic interpretation involving a random turn tug-of-war game, see [13, 3].

For a Lipschitz continuous function $u: \mathbb{R}^{n} \rightarrow \mathbb{R}$, let $w(x, t)$ and $v(x, t)$ be the unique viscosity solutions to the Hamilton-Jacobi flow equations

$$
\begin{cases}w_{t}-H(D w)=0 & \text { in } \left.\mathbb{R}^{n} \times\right] 0, \infty[ \\ w=u & \text { for } t=0\end{cases}
$$

and

$$
\begin{cases}v_{t}+H(D v)=0 & \text { in } \left.\mathbb{R}^{n} \times\right] 0, \infty[, \\ v=u & \text { for } t=0 .\end{cases}
$$

Our main result is the following:

Theorem 1.1. A Lipschitz continuous function $u: \mathbb{R}^{n} \rightarrow \mathbb{R}$ is a viscosity subsolution of $-A_{H}[\varphi]=0$ in $\mathbb{R}^{n}$ if and only if the function $t \mapsto w(x, t)$ in (1.2) is convex for all $x \in \mathbb{R}^{n}$. Similarly, $u$ is a viscosity supersolution of $-A_{H}[\varphi]=0$ in $\mathbb{R}^{n}$ if and only if the function $t \mapsto v(x, t)$ in (1.3) is concave for all $x \in \mathbb{R}^{n}$.

This characterization of subsolutions and supersolutions of (1.1) was conjectured by Barron, Evans and Jensen in [3]. Let us briefly recall its formal derivation. By differentiating (1.2) (assume $w$ is smooth) with respect to $t$ and $x_{k}$, we obtain

$$
w_{t t}-\sum_{i=1}^{n} H_{p_{i}}(D w) w_{x_{i} t}=0
$$

and

$$
w_{t x_{k}}-\sum_{i=1}^{n} H_{p_{i}}(D w) w_{x_{i} x_{k}}=0 .
$$

Substituting the second equation to the first yields

$$
w_{t t}-A_{H}[w]=0 .
$$

Using the fact that the flow (1.2) preserves subsolutions of (1.1), i.e., if $-A_{H}[u] \leq 0$ then $-A_{H}[w(\cdot, t)] \leq 0$ for all $t>0$ (see Lemma 2.1 below), we see that $-A_{H}[u] \leq 0$ 
implies $w_{t t} \geq 0$. Hence $t \mapsto w(x, t)$ is convex for every $x$. Conversely, the convexity of $t \mapsto w(x, t)$ together with (1.4) imply that $-A_{H}[w(\cdot, t)] \leq 0$ for all $t>0$ and hence also $-A_{H}[u] \leq 0$. The reasoning in the supersolution case is analogous, with the equation (1.2) being replaced by (1.3).

Already in the particular case of the infinity Laplacian $\Delta_{\infty} u=\sum u_{i} u_{j} u_{i j}$ Theorem 1.1 generalizes the known characterization of the subsolutions of the infinity Laplace equation $-\Delta_{\infty} u=0$ in terms of the convexity of the function

$$
t \mapsto \max _{|x-y|=t} u(y)=\max _{y \in B_{t}(x)} u(y)
$$

$c f$. [2, Section 4]. Indeed, this latter case correspond to the limiting case $H(p)=$ $|p|$, where the convexity assumptions on $H$ are no more valid. For this choice of Hamiltonian one can show that the functions $w$ and $v$ in (1.2) and (1.3) are precisely given as $w(x, t)=\max _{y \in B_{t}(x)} u(y)$ and $v(x, t)=\min _{y \in B_{t}(x)} u(y)$. We refer to [3] for more details.

Our idea for the proof of Theorem 1.1 is quite simple and starts with the observation that the evolution of a generalized cone function with non-negative slope under the backwards flow (1.2) is affine in time. More precisely, it is easy to check that in the radially symmetric case $H(p)=\frac{1}{2}|p|^{2}$, the unique viscosity solution to the equation $w_{t}-\frac{1}{2}|D w|^{2}=0$ with initial data $C(x)=a\left|x-x_{0}\right|+b, a \geq 0$, is given as $w(x, t)=C(x)+\frac{1}{2} a^{2} t$. For a general Hamiltonian $H$ the situation is analogous, see Proposition 2.5 below. Thus, in order to prove Theorem 1.1, it suffices to show that the comparison principle with respect to generalized cones that characterizes the viscosity subsolutions of (1.1) translates via (1.2) to comparison with respect to affine functions that in turn characterizes convexity, and vice versa. These two implications are proved in Sections 3 and 4.

Theorem 1.1 is stated for Lipschitz functions defined in the whole $\mathbb{R}^{n}$ mainly for the sake of clarity and transparency of the exposition. It will be clear from the proofs that the characterization is indeed local and applies to continuous functions defined on a subdomain $\Omega \subset \mathbb{R}^{n}$. We will elaborate on this in Section 5 .

\section{Preliminaries}

\subsection{Hamilton-Jacobi flows}

It is well-known (see e.g. [15]) that the functions $w$ and $v$ in (1.2) and (1.3), respectively, are given by the Hopf-Lax formulas

$$
\begin{aligned}
& w(x, t)=\sup _{y \in \mathbb{R}^{n}}\left(u(y)-t L\left(\frac{y-x}{t}\right)\right) \\
& v(x, t)=\inf _{y \in \mathbb{R}^{n}}\left(u(y)+t L\left(\frac{y-x}{t}\right)\right),
\end{aligned}
$$


where

$$
L(q):=\sup _{p \in \mathbb{R}^{n}}(p \cdot q-H(p))
$$

is the Legendre transform of $H$. Since $u$ is Lipschitz continuous, it is enough to take the sup and inf in (2.1) over those $y$ 's that satisfy

$$
L\left(\frac{y-x}{t}\right)<\operatorname{Lip}(u) \frac{|y-x|}{t} .
$$

By the superlinearity of $L$, this set is contained in the ball $B_{M t}(x)$ for some $M=$ $M(H, \operatorname{Lip}(u))>0$.

The Hamilton-Jacobi equations (1.2) and (1.3) appear frequently as smoothing devices in the theory of viscosity solutions. More precisely, the so-called inf- and sup-convolutions of $u$ (see $e . g$. [8, 12]) are nothing but the functions $x \mapsto w(x, \varepsilon)$ and $x \mapsto v(x, \varepsilon)$ given by (2.1) in the case $H(p)=L(p)=\frac{1}{2}|p|^{2}$. These convolutions provide semiconcave viscosity supersolutions and semiconvex subsolutions, respectively, for translation invariant equations.

Lemma 2.1. A uniformly continuous function $u: \mathbb{R}^{n} \rightarrow \mathbb{R}$ is a viscosity subsolution of $-A_{H}[\varphi]=0$ in $\mathbb{R}^{n}$ if and only if the function $x \mapsto w(x, t)$ is viscosity subsolution of $-A_{H}[\varphi]=0$ in $\mathbb{R}^{n}$ for every $t>0$.

Proof. This result is well-known, $c f .[12,2]$, but for the reader's convenience we sketch the proof. Suppose first that $-A_{H}[u] \leq 0$ (in the viscosity sense) and let $h(x)=w(x, t)$ for some fixed $t>0$. Since the Aronsson equation (1.1) is translation invariant, each of the functions

$$
x \mapsto u(x+t z)-t L(z), \quad z \in \mathbb{R}^{n}
$$

is a subsolution and clearly also uniformly continuous with the same modulus of continuity as $u$. Hence it follows from the definition of viscosity solutions that also

$$
h(x)=\sup _{z \in \mathbb{R}^{n}}(u(x+t z)-t L(z))
$$

is a viscosity subsolution of $-A_{H}[\varphi]=0$ in $\mathbb{R}^{n}$.

The converse implication follows from the stability of viscosity solutions and the fact that $w(x, t) \rightarrow u(x)$ locally uniformly as $t \rightarrow 0$.

\subsection{Generalized cone comparison}

The characterization of solutions in terms of the cone functions $x \mapsto a\left|x-x_{0}\right|+b$, discovered by Crandall, Evans and Gariepy in [7], is arguably the most important tool in the theory of the infinity Laplace equation. A similar characterization of the solutions of Aronsson equations of the form (1.1) has been recently obtained by Gariepy, Wang and Yu in [10] (see also [5, 16, 18]). Let us recall their main result. 
Definition 2.2. For $k \geq 0$, a generalized cone centered at the origin with slope $k$ is defined by

$$
C_{k}^{H}(x):=\max _{H(p)=k} x \cdot p .
$$

Definition 2.3. A continuous function $u: \mathbb{R}^{n} \rightarrow \mathbb{R}$ enjoys comparison with generalized cones from above if we have, for any bounded open set $V \subset \mathbb{R}^{n}, x_{0} \notin V$ and $k \geq 0$ that

$$
u(x) \leq u\left(x_{0}\right)+C_{k}^{H}\left(x-x_{0}\right) \quad \text { for all } x \in \partial V
$$

implies

$$
u(x) \leq u\left(x_{0}\right)+C_{k}^{H}\left(x-x_{0}\right) \quad \text { for all } x \in V .
$$

A continuous function $u: \mathbb{R}^{n} \rightarrow \mathbb{R}$ enjoys comparison with generalized cones from below if $-u$ enjoys comparison with generalized cones from above with respect to the Hamiltonian $\hat{H}(p):=H(-p)$.

Theorem 2.4 ([10]). A continuous function $u$ is a viscosity subsolution of the Aronsson equation $-A_{H}[u]=0$ if and only if $u$ enjoys comparison with generalized cones from above.

We next recall some basic properties of the generalized cone functions with $k>0$. Since $C_{k}^{H}$ is the supremum of linear functions $x \mapsto p \cdot x$, where $p \in H^{-1}(k)$, it is convex and Lipschitz continuous with the constant $R_{k}=\max \{|p|: H(p)=k\}$. Actually, by definition $C_{k}^{H}$ is the support function of the convex set

$$
K_{k}:=\{p: H(p) \leq k\} .
$$

Our assumptions on $H$ imply that the surface $\partial K_{k}$ is of class $C^{2}$ and uniformly convex, whence all its principal curvatures lie above a positive constant. This is known to imply $C_{k}^{H} \in C^{2}\left(\mathbb{R}^{n} \backslash\{0\}\right.$, and that the boundary of the dual convex set $K_{k}^{*}:=\left\{C_{k}^{H}(x) \leq 1\right\}$ is also of class $C^{2}$ and uniformly convex as well. By scaling the same holds for all non-trivial level sets of the function $C_{k}^{H}$. We refer $e$.g. to [14, Section 2.5] for the above facts.

Moreover, it is geometrically evident that for $x \neq 0$, the maximum in (2.3) is attained by the unique $p \in H^{-1}(k)$ for which

$$
\frac{D H(p)}{|D H(p)|}=\frac{x}{|x|} \text {. }
$$

Hence, if we denote by $Y_{k}: S^{n-1} \rightarrow H^{-1}(k)$ the inverse Gauss map defined by

$$
Y_{k}^{-1}(q)=\frac{D H(q)}{|D H(q)|} \quad \text { for } q \in H^{-1}(k),
$$

it follows that $C_{k}^{H}(x)=x \cdot Y_{k}\left(\frac{x}{|x|}\right)$ for $x \neq 0$ and, by the discussion in the preceding paragraph (see also [10]) we have that $Y_{k} \in C^{1}\left(S^{n-1}\right)$. Furthermore,

$$
D C_{k}^{H}(x)=Y_{k}\left(\frac{x}{|x|}\right) \quad \text { for } x \neq 0 .
$$


Indeed, by definition, $C_{k}^{H}(y)-p \cdot y \geq 0$ for all $y \in \mathbb{R}^{n}$ and for any fixed $p \in$ $H^{-1}(k)$ (and hence for $p=Y_{k}\left(\frac{x}{|x|}\right)$ ). On the other hand, the equality $C_{k}^{H}(x)-$ $Y_{k}\left(\frac{x}{|x|}\right) \cdot x=0$ holds for $x \neq 0$ by the definition of $Y_{k}\left(\frac{x}{|x|}\right)$, and thus the mapping $y \mapsto C_{k}^{H}(y)-Y_{k}\left(\frac{x}{|x|}\right) \cdot y$ attains its minimum at $x$. The relation (2.5) now follows. The proof of Theorem 1.1 relies strongly on the following observation:

Proposition 2.5. Let $\Psi(x, t)$ be the unique viscosity solution to

$$
\begin{cases}\Psi_{t}-H(D \Psi)=0 & \text { in } \left.\mathbb{R}^{n} \times\right] 0, \infty[ \\ \Psi=C_{k}^{H} & \text { for } t=0 .\end{cases}
$$

Then $\Psi(x, t)=C_{k}^{H}(x)+k t$. In particular, $t \mapsto \Psi(x, t)$ is affine for all $x \in \mathbb{R}^{n}$. If $x=0$ and $t>0$, then in the representation

$$
\Psi(x, t)=\max _{y \in \mathbb{R}^{n}}\left(C_{k}^{H}(y)-t L\left(\frac{y-x}{t}\right)\right),
$$

the maximum is achieved exactly at all points $y$ that satisfy $y \in t S_{k}$, where

$$
S_{k}:=D H\left(H^{-1}(k)\right) \text {. }
$$

The surface $S_{k}$ is the boundary of a bounded domain that contains the origin.

Proof. By the above discussion, the function $(x, t) \mapsto C_{k}^{H}(x)+k t$ satisfies the equation $f_{t}-H(D f)=0$ a.e. and is (semi)convex, and therefore it is the unique viscosity solution to (2.6). See e.g. [9, Sections 3.3 and 10.3].

In order to prove the last statement of the theorem we may assume that $t=1$, as the general case is readily obtained by scaling. We shall determine the zeroset of the derivative of the difference $F(y):=C_{k}^{H}(y)-L(y)$. Observe that $F$ is differentiable outside the origin and assume first that $z \neq 0$ satisfies $H(z)=k$. The 0-homogeneity of the function $D C_{k}^{H}$ and the formulae (2.4) and (2.5) yield

$$
D C_{k}^{H}(D H(z))=D C_{k}^{H}\left(\frac{D H(z)}{|D H(z)|}\right)=Y_{k}\left(\frac{D H(z)}{|D H(z)|}\right)=z .
$$

By substituting above $z=D L(y)$ and applying the relation $D H \circ D L=I$ (see e.g. [17, Chapter 51]), we see that the condition $H(D L(y))=k$ implies $D C_{k}^{H}(y)=$ $D L(y)$, or in other words $D F(y)=0$. Conversely, if $D F(y)=0$ and $y \neq 0$, then

$$
H(D L(y))=H\left(D C_{k}^{H}(y)\right)=H\left(Y_{k}\left(\frac{y}{|y|}\right)\right)=k .
$$

Using again the fact $D H \circ D L=I$ we see that the condition $H(D L(y))=k$ is equivalent to $y \in D H\left(H^{-1}(k)\right)$. We have thus shown that $S_{k}=\{y \neq 0$ : $D F(y)=0\}$. Since the function $F$ attains its maximum outside origin, and the compact and connected surface $S_{k}$ is locally Lipschitz, we deduce that $F$ attains its maximum exactly at the points $y \in S$. The remaining statements are obvious. 
Remark 2.6. Due to the semiconvexity requirement for the solution of (2.6), Proposition 2.5 does not hold for cones with negative slope. See [9, Section 3.3] for an explicit counterexample.

The following technical lemma is needed later on.

Lemma 2.7. (i) We have

$$
C_{k}^{H}(y+x) \leq C_{k}^{H}(x)+C_{k}^{H}(y) \quad \text { for any } x, y \in \mathbb{R}^{n} .
$$

(ii) Let $k>0$ and $x \in \mathbb{R}^{n} \backslash\{0\}$. Then

$$
D^{2} C_{k}^{H}(x) \xi \cdot \xi \geq 0
$$

for all $\xi \neq 0$ and the equality holds if and only if $\xi$ is parallel to $x$.

Proof. (i) As noted in [10], this follows immediately from the definition of $C_{k}^{H}$ :

$$
\begin{aligned}
C_{k}^{H}(y+x)=\max _{H(p)=k}(x+y) \cdot p & \leq \max _{H(p)=k} x \cdot p+\max _{H(p)=k} y \cdot p \\
& =C_{k}^{H}(x)+C_{k}^{H}(y) .
\end{aligned}
$$

(ii) The stated inequality follows directly from the convexity of the function $C_{k}^{H}$. In order to treat the case of equality, we first show that there is a positive constant $\beta$ such that

$$
D^{2} C_{k}^{H}(x) \xi \cdot \xi \geq \beta|\xi|^{2} \text { for any } \xi \text { with } \xi \cdot D C_{k}(x)=0 .
$$

Denote $p=D C_{k}(x)$ and $T=\{\xi: p \cdot \xi=0\}$. The surface $U=\left\{y: C_{k}^{H}(y)=\right.$ $\left.C_{k}^{H}(x)\right\}=C_{k}^{H}(x) K^{*}$ is uniformly convex and the hyperplane $T+x$ is tangent to $U$ at the point $x$. Hence the map $\xi \mapsto \operatorname{dist}(x+\xi, U)$, where $\xi \in T$, is uniformly convex. A fortiori, if $\xi \in T$ is small enough we may use the homogeneity of $C_{k}^{H}$ to estimate

$$
C_{k}^{H}(x+\xi) \geq b_{1} \operatorname{dist}(\xi+x, U)+C_{k}^{H}(x) \geq b_{2}|\xi|^{2}+C_{k}^{H}(x),
$$

where $b_{2}>0$. This immediately yields inequality (2.9).

According to (2.9) the rank of the matrix $D^{2} C_{k}^{H}(x)$ is at least $n-1$, and by noting that $D^{2} C_{k}^{H}(x) x \cdot x=0$ we see that it is exactly $n-1$. These observations yield the second part of the lemma.

\section{Sufficiency}

Theorem 2.4 already implies that if $u$ is not a viscosity subsolution of $-A_{H}[u]=0$, then the comparison with respect to generalized cones from above fails. However, in the proof of Theorem 1.1 we will need a refined version of this fact. 
Proposition 3.1. Assume that $\Omega \subset \mathbb{R}^{n}$ is a domain and that the function $u \in C(\Omega)$ is not a viscosity subsolution of $-A_{H}[u]=0$ in $\Omega$. Then there exists $\hat{x} \in \Omega$ and $k>$ 0 with the following property: for all $\delta \in\left(0, \frac{1}{2} \operatorname{dist}(\hat{x}, \partial \Omega)\right)$ and $N \geq 1$ there exists $\varepsilon>0$, a point $x_{0} \in B(\hat{x}, \varepsilon)$, and a generalized cone $C(x)=C_{k}^{H}\left(x-x_{0}\right)+u\left(x_{0}\right)$ such that $0<N \varepsilon<\delta$ and, moreover

1. the set $D:=\left\{x \in B_{N \varepsilon}\left(x_{0}\right): u(x)>C(x)\right\}$ is non-empty and contained in $B_{\varepsilon}\left(x_{0}\right)$. Moreover, $x_{0} \in \partial D$.

2. $\sup _{B_{N \varepsilon}\left(x_{0}\right)}(u-C) \leq \varepsilon$.

Proof. Since $u$ is not a viscosity subsolution of $-A_{H}[u]=0$, there exists $\varphi \in$ $C^{2}(\Omega)$ and $\hat{x} \in \Omega$ such that $0=u(\hat{x})-\varphi(\hat{x})>u(x)-\varphi(x)$ for $x \in \Omega \backslash\{\hat{x}\}$ and

$$
\left(D^{2} \varphi(\hat{x}) D H(D \varphi(\hat{x}))\right) \cdot D H(D \varphi(\hat{x}))<0 .
$$

Assume that $\delta \in\left(0, \frac{1}{3} \operatorname{dist}(\hat{x}, \partial \Omega)\right)$ and $N \geq 1$ are given. In order to prove our claim, it suffices to show that there exists $a>0, b \in \mathbb{R}$ and $y_{0} \in B(\hat{x}, \delta / 3 N)$ such that the function $\varphi-\left(C_{a}^{H}\left(x-y_{0}\right)+b\right)$ has a strict local zero maximum at $\hat{x}$. Indeed, if this is the case, by first decreasing $b$ slightly enough and then translating the vertex $y_{0}$ along the segment $\left[y_{0}, \hat{x}\right)$ to a point $x_{0}$ at which $u$ and the dropped cone agree (and adjusting $b$ again suitably), we obtain (1) and (2) with an appropriate $\varepsilon>0$. The translation preserves the required other properties of the cone, as is easily seen with the aid of Lemma 2.7 (i).

Let $p=D \varphi(\hat{x})$, whence (3.1) shows that $p \neq 0$. Denote $a=H(p)>0$ and $z_{0}=\frac{D H(p)}{|D H(p)|} \neq 0$. For $z=\lambda z_{0}$, where the constant $\lambda>0$ small enough will be chosen below, it holds that $C_{a}^{H}(z)=z \cdot p$ and $D C_{a}^{H}(z)=p, c f$. (2.5). We define

$$
C_{0}(x)=C_{a}^{H}\left(x-y_{0}\right)+b,
$$

where $a=H(p)=H(D \varphi(\hat{x})), y_{0}=\hat{x}-z$ and $b=\varphi(\hat{x})-C_{a}^{H}\left(\hat{x}-y_{0}\right)$. Then $C_{0}(\hat{x})=\varphi(\hat{x})$ and $D C_{0}(\hat{x})=D C_{a}^{H}(z)=D \varphi(\hat{x})$, and thus is suffices to check the strict matrix inequality $D^{2} C_{0}(\hat{x})>D^{2} \varphi(\hat{x})$.

Let us denote $S^{n-1}=\{y:|y|=1\}$ and

$$
A=\left\{\xi \in S^{n-1}: D^{2} \varphi(\hat{x}) \xi \cdot \xi \geq 0\right\} .
$$

Set $\alpha:=\sup _{\xi \in S^{n-1}} D^{2} \varphi(\hat{x}) \xi \cdot \xi$. Observe that (3.1) implies that $z_{0}$ is not in the closure of $A$. Hence, by Lemma 2.7 (ii), the quantity

$$
c:=\inf _{\xi \in A} D^{2} C_{a}^{H}\left(z_{0}\right) \xi \cdot \xi
$$

is positive. Thus, by scaling and the (-1)-homogeneity of $D^{2} C_{a}^{H}$ we obtain that

$$
D^{2} C_{0}(\hat{x}) \xi \cdot \xi=\lambda^{-1} D^{2} C_{a}^{H}\left(z_{0}\right) \xi \cdot \xi>D^{2} \varphi(\hat{x}) \xi \cdot \xi \quad \text { for all } \xi \neq 0
$$

as soon as $\lambda<c / \alpha$. In other words, then $D^{2} C_{0}(\hat{x})>D^{2} \varphi(\hat{x})$. This completes the proof. 
Let us now prove the sufficiency part of Theorem 1.1. Let $u: \mathbb{R}^{n} \rightarrow \mathbb{R}$ be Lipschitz continuous and let $w \in C\left(\mathbb{R}^{n} \times[0, \infty[)\right.$ be the unique viscosity solution to

$$
\begin{cases}w_{t}-H(D w)=0 & \text { in } \left.\mathbb{R}^{n} \times\right] 0, \infty[ \\ w=u & \text { for } t=0 .\end{cases}
$$

If $u$ is not a subsolution of $-A_{H}[u]=0$, we choose according to Proposition 3.1 a point $\hat{x}$ and a constant $k>0$ with the stated properties. In particular, if $R_{k}:=$ $\max \{|p|: H(p)=k\}$ is the Lipschitz constant of $C_{k}^{H}$ and $M$ is determined by (2.2), we may apply the choices $\delta=1$ and

$$
N=\frac{2 M\left(1+R_{k}\right)}{k}+1
$$

to infer the existence of an $\varepsilon \in(0,1 / N)$ and a cone $C(x)=C_{k}^{H}\left(x-x_{0}\right)+u\left(x_{0}\right)$ such that (1) and (2) of the Proposition hold. Without loss of generality, we may assume that $x_{0}=0$ and $C(0)=u(0)=0$. Let $\Psi \in C\left(\mathbb{R}^{n} \times[0, \infty[)\right.$ be the unique viscosity solution to

$$
\begin{cases}\Psi_{t}-H(D \Psi)=0 & \text { in } \left.\mathbb{R}^{n} \times\right] 0, \infty[ \\ \Psi=C & \text { for } t=0 .\end{cases}
$$

By Proposition 3.1 (1), we can find arbitrarily small $t>0$ for which there exist $y_{t} \in \mathbb{R}^{n}$ such that $y_{t} \in t S_{k}$, i.e. $H\left(D L\left(\frac{y_{t}}{t}\right)\right)=k$, and $u\left(y_{t}\right)>C_{k}^{H}\left(y_{t}\right)$. Thus, for such $t$ 's, we obtain using Proposition 2.5 that

$$
\Psi(0, t)=C_{k}^{H}\left(y_{t}\right)-t L\left(\frac{y_{t}}{t}\right)<u\left(y_{t}\right)-t L\left(\frac{y_{t}}{t}\right) \leq w(0, t) .
$$

On the other hand, by the choice of $N$ we see that

$$
\Psi\left(0, \frac{N \varepsilon}{M}\right) \geq w\left(0, \frac{N \varepsilon}{M}\right) .
$$

Indeed, if $t=\frac{N \varepsilon}{M}$ we have $B_{M t}(0)=B_{N \varepsilon}(0)$ and thus, in view of Proposition 3.1, the reverse inequality $\Psi(0, t)<w(0, t)$ can hold only if there exists $z \in B_{\varepsilon}(0)$ such that $u(z)>C_{k}^{H}(z)$ and $u(z)-t L\left(\frac{z}{t}\right)>\Psi(0, t)$. Since $u(z) \leq C_{k}^{H}(z)+\varepsilon$ and $\Psi(0, t)=k t$, we must then have

$$
C_{k}^{H}(z)+\varepsilon-t L\left(\frac{z}{t}\right)>k t .
$$

But this is impossible, because

$$
C_{k}^{H}(z)+\varepsilon-t L\left(\frac{z}{t}\right) \leq C_{k}^{H}(z)+\varepsilon \leq\left(1+R_{k}\right) \varepsilon \leq k \frac{N \varepsilon}{2 M} \leq k t
$$

by the choice of $N$. By combining (3.4) and (3.5) with the equality $\Psi(0,0)=$ $C(0)=u(0)=w(0,0)$, and recalling that $t \mapsto \Psi(0, t)$ is linear, we conclude that $t \mapsto w(0, t)$ is not convex. 
Remark 3.2. Observe that in the above proof we consider only $u$ in the neighborhood $B(\hat{x}, \delta)$. Moreover, the time parameter varies over the interval $t \in[0, \delta / M]$, where $M$ depends only on $H$ and $u$, but not on $\delta$. This observation will be needed later on in Section 5.

\section{Necessity}

We now prove the necessity part of Theorem 1.1. For a viscosity subsolution $u$ of $-A_{H}[\varphi]=0$, define $w(x, 0)=u(x)$ for $x \in \mathbb{R}^{n}$ and denote

$$
w(x, t)=\sup _{y \in \mathbb{R}^{n}}\left(u(y)-t L\left(\frac{y-x}{t}\right)\right) \quad \text { for } t>0 \text { and } x \in \mathbb{R}^{n} .
$$

Suppose that $f(t):=w(0, t)$ is not convex. Then there exists $0 \leq t_{1}<t_{2}$ and an affine function $l(t)=a t+b$ such that

$$
l\left(t_{i}\right)=f\left(t_{i}\right), i=1,2, \quad \text { and } \quad l(t)<f(t) \quad \text { for all } t_{1}<t<t_{2} .
$$

By the definition of $f$ and the semigroup property of the flow we see that $f$ is non-decreasing, whence we may assume without loss of generality that $a>0$. Moreover, by Lemma 2.1 and by invoking again the semigroup property, we may assume that $t_{1}=0$ and thus $b=u(0)$.

Let $C(x)=C_{a}^{H}(x)+u(0)$ and let $\Psi(x, t)$ be the unique solution to

$$
\begin{cases}\Psi_{t}-H(D \Psi)=0 & \text { in } \left.\mathbb{R}^{n} \times\right] 0, \infty[ \\ \Psi=C & \text { for } t=0\end{cases}
$$

given by the Hopf-Lax formula

$$
\Psi(x, t)=\sup _{y \in \mathbb{R}^{n}}\left(C(y)-t L\left(\frac{y-x}{t}\right)\right) .
$$

By Proposition 2.5, $\Psi(x, t)=C_{a}^{H}(x)+u(0)+a t$. In particular, $\Psi(0, t)=l(t)$ for all $t \geq 0$.

For $0<t<t_{2}, \Psi(0, t)<w(0, t)$ by (4.1), and by (2.2) this is possible only if

$$
\{x: u(x)>C(x)\} \cap B(0, t) \neq \varnothing \quad \text { for all } 0<t<t_{2} .
$$

On the other hand, by Proposition $2.5, \Psi\left(0, t_{2}\right)=w\left(0, t_{2}\right)$ implies that $u \leq C$ on the "sphere" $t_{2} S_{a}$. We have thus shown that the generalized cone comparison principle of Definition 2.3 is violated, contrary to the assumption that $u$ is a viscosity subsolution of $-A_{H}[\varphi]=0$. 


\section{Characterization in subdomains}

Throughout the preceding sections, it was assumed that $u$ is a Lipschitz function defined in the whole $\mathbb{R}^{n}$. This rendered the proofs quite transparent, but, in view of the existing literature on the Aronsson equations, it is also natural to try to consider more general situations. Fortunately, since all our arguments are local in nature, it turns out that we need neither the Lipschitz continuity nor the fact that the domain is the entire $\mathbb{R}^{n}$ to obtain a characterization for the sub- and supersolutions.

Let $\Omega \subset \mathbb{R}^{n}$ be a bounded domain and $u: \Omega \rightarrow \mathbb{R}$ a continuous and bounded function. ${ }^{1}$ We define

$$
w(x, t)=\sup _{y \in \Omega}\left(u(y)-t L\left(\frac{y-x}{t}\right)\right),
$$

and notice that for a fixed $x \in \Omega$ it suffices to take the supremum over those $y$ 's that satisfy

$$
t L\left(\frac{y-x}{t}\right) \leq 2\|u\|_{\infty} .
$$

Since $L$ is superlinear, there exists a non-decreasing, continuous function $M(t)$ such that this set is contained in the ball $B_{M(t)}(x)$. Hence there exists a continuous function $r: \Omega \rightarrow] 0, \infty[$ such that for all $0<t \leq r(x)$ the points $y$ for which (5.2) holds are compactly contained in $\Omega$.

Remark 5.1. Observe that in the case of a bounded domain, instead of using the Hamilton-Jacobi equation (1.2), we defined the function $w$ directly by the HopfLax formula (5.1). However, since the proof of Lemma 2.1 is based on the use of Hopf-Lax formula, and also the semigroup property of $\{w(x, t)\}_{t \geq 0}$ can be proved without invoking (1.2), see [9, Section 3.3], we have the same auxiliary results at our disposal as in the situation of Theorem 1.1. Actually, it turns out that $w_{t}=H(D w)$ in the viscosity sense at least in $\{(x, t): x \in \Omega, 0<t<r(x)\}, c f$. [9], but we do not need this fact.

We now claim that:

Theorem 5.2. A continuous and bounded function $u: \Omega \rightarrow \mathbb{R}$ is a viscosity subsolution of the Aronsson equation $-A_{H}[\varphi]=0$ in $\Omega$ if and only if $t \mapsto w(x, t)$ (where $w$ is defined by (5.1)) is convex in the interval $[0, r(x)]$.

The proof of Theorem 5.2 is essentially the same as that of Theorem 1.1 and we only indicate the necessary additional observations, retaining the notation and conventions used before. As regards sufficiency, assume that the bounded function $u \in C(\Omega)$ is not a subsolution. Then, by an analogue of Lemma 2.1 there is a subdomain $\Omega^{\prime} \subset \Omega$ and an arbitrary small time instant $t_{0}>0$ such that the function $h(x)=u\left(x, t_{0}\right)$ is not a subsolution in $\Omega^{\prime}$. Moreover, by decreasing $\Omega^{\prime}$ if needed, we may assume that $h$ is Lipschitz continuous in $\Omega^{\prime}$.

${ }^{1}$ Alternatively, we could just assume that $u$ is continuous and work in a subdomain of $\Omega$. 
One may now proceed exactly as before: we first choose a point $\hat{x} \in \Omega^{\prime}$ with the properties stated in Proposition 3.1. According to Remark 3.2, for small enough $\delta>0$ the reasoning in Section 3 takes place in the neighborhood $(y, t) \in B(\tilde{x}, \delta) \times$ $[0, \delta / M]$, where $\tilde{x} \in \Omega$ and $M=M\left(H, \operatorname{Lip}(h), \Omega^{\prime}\right)$ are independent of $\delta$. As $\delta>0$ can be taken arbitrarily small, it is clear that the argument used in case $\Omega=\mathbb{R}^{n}$ carries through unchanged.

Concerning the proof of the necessity, we note that the part of the argument that may cause problems is the derivation of the inequality $u \leq C$ on the "sphere" $t_{2} S_{a}$. In fact, it may very well happen that this set is not contained in $\Omega$ and then $u$ is not even defined on it. To overcome this difficulty, we use a one dimensional version of Proposition 3.1 (which, of course, can also be proved directly):

Lemma 5.3. Suppose that a continuous non-decreasing function $f:[0, d] \rightarrow \mathbb{R}$ is not convex. Then there exists $\hat{x} \in] 0, d[$, and $a>0$ with the following properties: for all small enough $\delta>0$ and $N>1$ there is $\varepsilon>0$ satisfying $2 N \varepsilon<\delta$, a point $x_{0}$ with $\left|x_{0}-\hat{x}\right|<\varepsilon$, and an affine function $l(x)=a x+b, a>0$, such that

1. the set $D:=\left\{x \in B_{N \varepsilon}\left(x_{0}\right): f(x)>l(x)\right\}$ is non-empty and contained in $B_{\varepsilon}\left(x_{0}\right)$. Moreover, $x_{0} \in \partial D$.

2. $\sup _{B_{N \varepsilon}\left(x_{0}\right)}(f-l) \leq \varepsilon$.

Thus, if we follow the proof of necessity in Section 4 we may assume that both $t_{1}>0$ and $t_{2}-t_{1}>0$ are as small as we wish, and, moreover, that the slope $a>0$ does not depend on the choice of $t_{1}, t_{2}$. After these preliminary observations it is clear that the argument can be completed exactly as before.

\section{References}

[1] G. AronsSon, Extension of functions satisfying Lipschitz conditions, Ark. Mat. 6 (1967), 551-561.

[2] G. Aronsson, M. G. Crandall and P. JuUtinen, A tour of the theory of absolutely minimizing functions, Bull. Amer. Math. Soc. (N.S.) 41 (2004), 439-505.

[3] E. N. BARron, L. C. Evans and R. R. JENSEN, The infinity Laplacian, Aronsson's equation and their generalizations, preprint.

[4] E. N. BARRON, R. R. JENSEN and C. Y. WANG, The Euler equation and absolute minimizers of $L^{\infty}$ functionals, Arch. Rational Mech. Anal. 157 (2001), 255-283.

[5] T. CHAMPION and L. DE PASCALE, A principle of comparison with distance functions for absolute minimizers, J. Convex Anal., to appear.

[6] M. G. CRAndAll, An efficient derivation of the Aronsson equation, Arch. Rational Mech. Anal. 167 (2003), 271-279.

[7] M. G. Crandall, L. C. Evans and R. F. Gariepy, Optimal Lipschitz extensions and the infinity Laplacian, Calc. Var. Partial Differential Equations 13 (2001), 123-139.

[8] M. G. CRANDALL, H. IshiI and P.-L. LiONS, User's guide to viscosity solutions of second order partial differential equations, Bull. Amer. Math. Soc. (N.S.) 27 (1992), 1-67.

[9] L. C. Evans, "Partial Differential Equations", Graduate Studies in Mathematics, Vol. 19, American Mathematical Society, Providence, RI, 1998.

[10] R. GARIEPY, C. Y. WANG and Y. YU, Generalized cone comparison principle for viscosity solutions of the Aronsson equation and absolute minimizers, Comm. Partial Differential Equations 31 (2006), 1027-1046. 
[11] R. R. JENSEN, Uniqueness of Lipschitz extensions: minimizing the sup norm of the gradient, Arch. Rational Mech. Anal. 123 (1993), 51-74.

[12] R. R. JENSEN, P.-L. Lions and P. E. Souganidis, A uniqueness result for viscosity solutions of second order fully nonlinear partial differential equations, Proc. Amer. Math. Soc. 102 (1988), 975-978.

[13] Y. Peres, O. Schramm, S. ShefField and D. B. Wilson, Tug-of-war and the infinity Laplacian, preprint (available at http://arxiv.org/archive/math)

[14] R. SchneIDER, "Convex Bodies: The Brunn-Minkowski Theory", Cambridge University Press, 1993.

[15] T. StRömberg, The Hopf-Lax formula gives the unique viscosity solution, Differential Integral Equations 15 (2002), 47-52.

[16] C. WANG and Y. YU, $C^{1}$-regularity of the Aronsson equation in $\mathbb{R}^{2}$, Ann. Inst. H. Poincaré Anal. Non Linéaire, to appear.

[17] E. ZEIDLER, "Nonlinear Functional Analysis and its Applications. III. Variational Methods and Optimization", Springer-Verlag, New York, 1985.

[18] Y. YU $L^{\infty}$ variational problems and Aronsson equations, Arch. Ration. Mech. Anal. 182 (2006), 153-180.

Department of Mathematics and Statistics University of Jyväskylä

P.O. Box 35

FIN-40014 Jyväskylä, Finland

peanju@maths.jyu.fi

saksman@maths.jyu.fi 